\title{
Interação genótipo $\times$ ambiente e estimativas de parâmetros genéticos de características ponderais de bovinos Tabapuã
}

\author{
[Genotype by environment interaction and genetic parameter estimates for growth traits of Tabapuã cattle] \\ A.B. Fridrich ${ }^{1}$, M.A. Silva ${ }^{1}$, D. Fridrich ${ }^{2}$, G.S.S. Corrêa ${ }^{1}$, L.O.C. Silva ${ }^{3}$, E.S. Sakaguti ${ }^{4}$, \\ I.C. Ferreira ${ }^{1}$, B.D. Valente ${ }^{1}$ \\ ${ }^{1}$ Escola de Veterinária da UFMG \\ Caixa Postal 567 \\ 30123-970 - Belo Horizonte, MG \\ ${ }^{2}$ Departamento de Medicina Veterinária - CAV - UDESC - Lages, SC \\ ${ }^{3}$ EMBRAPA Gado de Corte - CNPGC - Campo Grande, MS \\ ${ }^{4}$ Departamento de Zootecnia - CCA - UEM - Maringá, PR
}

\begin{abstract}
RESUMO
Dados de pesos aos 205 (P205) e 365 (P365) dias de idade, de 28.946 animais Tabapuã, provenientes de 152 fazendas dos diversos estados brasileiros, nascidos no período de 1976 a 1995, foram utilizados nesta análise. Foram avaliadas as interações genótipo-ambiente, bem como estimadas herdabilidades direta e materna pelo método de máxima verossimilhança restrita em modelo estatístico, que incluiu efeitos fixos de grupo contemporâneo, idade da vaca ao parto (covariável) e efeitos aleatórios genéticos direto e materno. As estimativas de herdabilidade direta e materna para P205 nas regiões Sul (R1), Sudeste (R2), Centro-Oeste (R3) e Nordeste (R4) foram: 0,02 e 0,31 (R1), 0,17 e 0,19 (R2), 0,20 e 0,09 (R3) e 0,06 e 0,16 (R4). Para P365, foram 0,05 e 0,03 (R1), 0,20 e 0,03 (R2), 0,51 e 0,62 (R3) e 0,15 e 0,05 (R4). As correlações genéticas encontradas para as características P205 e P365, ambas consideradas características distintas nas regiões R1, R2, R3 e R4, foram: 1,00 e $0,99,0,84$ e $0,99,-0,86$ e - $0,73,0,98$ e $0,93,0,51$ e 0,45, 1,00 e 0,12 para R1/R2, R1/R3, R1/R4, R2/R3, R2/R4 e R3/R4, respectivamente. Esses resultados indicam que, na desmama (P205), o efeito da interação genótipo $\mathrm{x}$ ambiente foi observado somente nas combinações que envolveram a região Nordeste (R4) e as regiões Sul (R1) e Sudeste (R2). Para pesos pós-desmama (P365), o efeito dessa interação foi evidenciado em todas as combinações que incluíram a região Nordeste.
\end{abstract}

Palavras-chave: bovino, Tabapuã, característica ponderal, herdabilidade direta e materna, interação genótipoambiente

\begin{abstract}
Body weight records at 205 (205BW) and 365 (365BW) days of age of 28,946 Tabapuã animals born during the 1976-1995 period from 152 Tabapuã herds of several states of Brazil, were used to evaluate genotype by environment interactions and to estimate genetic and maternal heritability by restricted maximum likelihood methodology. The statistical model included the fixed effects of contemporary group and age of cow (covariate), and the random additive genetic and maternal effects. Maternal and genetic heritability estimates for BW205 considered as different traits in each of the South (R1), Southeast (R2), Central West (R3), Northeast $(R 4)$ regions were .02 and $.31, .17$ and $.19, .20$ and .09 and .06 and .16 , respectively, and for
\end{abstract}

Recebido para publicação em 19 de fevereiro de 2003

Recebido para publicação, após modificações, em 10 de novembro de 2004

E-mail: martinho@vet.ufmg.br 
BW365 they were .05 and .03 (R1), .20 and .03 (R2), .51 and .62 (R3) and .15 and .05 (R4). The genetic correlation for 205BW and $365 \mathrm{BW}$ both considered as different traits in each of the South (R1), Southeast (R2), Central West (R3), Northeast (R4) regions were, respectively, 1.00 and .99, .84 and .99, -.86 and -.73, .98 and .93, .51 and .45, 1.00 and .12 for BW205 and $B W 365$ in R1/R2, R1/R3, R1/R4, R2/R3, R2/R4 e R3/R4, respectively, indicating a significant genotype by environment interaction for $205 B W$ only for the combination between Northeast and the South and Southeast regions. For the 365BW there was a significant genotype by environment interaction for all combinations involving the Northeast region.

Keywords: bovine, Tabapuã breed, direct and maternal heritability, genotype-environment interaction, growth trait

\section{INTRODUÇÃO}

A magnitude da interação genótipo-ambiente e a estimação de componentes de (co)variância são importantes para os programas de melhoramento genético dos animais domésticos. Estudos da interação genótipo-ambiente iniciaram-se por volta do século XX e, em geral, visavam estabelecer se a seleção deveria ser praticada no ambiente em que o animal seria criado ou em ambiente melhorado, para que pudesse expressar o máximo do seu potencial genético.

Em 1947, Hammond propôs que os animais deveriam ser selecionados nos melhores ambientes, para expressar melhor os genes de interesse. Já em 1952, Falconer sugeriu que o conjunto de genes responsáveis pela expressão de determinada característica podia variar em função do ambiente de criação. A diversificação de climas e manejo no Brasil resulta em grande variação na produção nos rebanhos.

A difusão da prática de inseminação artificial aumentou a possibilidade de reprodutores terem maior número de progênies em diferentes rebanhos de diversas regiões brasileiras com níveis de manejo também diferentes. Se o reprodutor tiver progênies em muitos ambientes, qualquer efeito da interação poderá ser confundido com a estimativa do mérito genético, limitando tanto a capacidade de estimar as diferenças entre touros como a efetividade da comparação de touros entre rebanhos (Buchanan e Nielsen, 1979).

A correta avaliação do genótipo por intermédio de seu fenótipo constitui a base de todos os programas de melhoramento genético. Na presença de interação, entretanto, essa avaliação poderá variar de um ambiente para outro, resultando em mudança na ordem de classificação (rank/ posto) dos genótipos ou, ainda, na magnitude de suas diferenças.

Neste trabalho, avaliou-se a interação genótipo $\mathrm{x}$ ambiente em características ponderais de bovinos de corte Tabapuã bem como estimaram-se seus componentes genéticos.

\section{MATERIAL E MÉTODOS}

As informações utilizadas neste estudo são de animais Tabapuã, nascidos entre 1976 e 1995, provenientes de 152 fazendas dos diversos estados brasileiros, incluídos no Controle de Desenvolvimento Ponderal da Associação Brasileira de Criadores de Zebu (ABCZ) e cedidos pela Embrapa Gado de Corte, localizada em Campo Grande, MS.

As características estudadas foram os pesos do animal aos 205 (P205) e 365 (P365) dias de idade. Os pesos foram padronizados por interpolação, e o intervalo considerado entre as pesagens foi, no máximo, de 40 dias, de acordo com o sistema de avaliação genética adotado pelo Controle de Desenvolvimento Ponderal da ABCZ.

Três arquivos foram formados para as análises. Os dois primeiros continham os dados do animal, mãe, grupo contemporâneo, idade da vaca ao parto e os pesos aos 205 e 365 dias de idade, considerados como características distintas em cada região, e o terceiro, os dados recodificados do animal, do pai e da mãe. 
Os arquivos, para avaliar o efeito da interação genótipo $\mathrm{x}$ ambiente sobre as características analisadas nas diversas regiões brasileiras, foram constituídos apenas com informações de animais criados exclusivamente no pasto e com suplementação mineral. Os estados foram agrupados em cinco regiões distintas: 1-Sul, 2Sudeste, 3-Centro-Oeste, 4-Norte e 5-Nordeste.

Foram consideradas duas estações de nascimento, de acordo com as épocas das águas e das secas das regiões. Foram também definidos os "touros de conexão", isto é, touros com número mínimo de duas progênies em cada uma de três ou mais regiões. Em seguida, os grupos contemporâneos (GC) foram formados por ano de nascimento, fazenda, estação de nascimento e sexo. As regiões que apresentavam número de $\mathrm{GC}$ menor do que três foram eliminadas.

As análises estatísticas dos pesos ajustados às idades-padrão foram feitas com o auxílio do procedimento generalized linear models (GLM), do pacote estatístico SAS (Statistical..., 1997). Os arquivos formados foram constituídos de 19.482 e 15.848 animais. Na Tab. 1 encontram-se algumas estatísticas descritivas das características da amostra estudada.

Tabela 1. Estatística descritiva dos arquivos com informações de pesos aos 205 e 365 dias de idade nas diversas regiões estudadas

\begin{tabular}{lcccc}
\hline Item & Sul & Sudeste & Centro-Oeste & Nordeste \\
\hline $\mathrm{N}^{\circ}$ animais - P205 & 799 & 13.273 & 479 & 2.716 \\
$\mathrm{~N}^{\circ}$ animais $-\mathrm{P} 365$ & 722 & 10.388 & 431 & 2.255 \\
$\mathrm{~N}^{\circ}$ animais na A & -1 & 28.946 & 28.946 & 28.946 \\
Média \pm DP arquivo 2 & $167,83 \pm 24,3$ & $172,69 \pm 24,6$ & $169,10 \pm 23,3$ & $171,70 \pm 30,6$ \\
Média \pm DP arquivo 3 & $215,18 \pm 37,7$ & $223,10 \pm 36,61$ & $207,41 \pm 30,3$ & $225,64 \pm 38,3$ \\
$\mathrm{CV} \%$ - P205 & 14,48 & 14,30 & 13,79 & 17,84 \\
$\mathrm{CV} \%$ - P365 & 17,53 & 16,41 & 14,60 & 17,01 \\
\hline
\end{tabular}

$\mathrm{A}^{-1}=$ matriz de numeradores dos coeficientes de parentesco é igual para todas os arquivos.

$\mathrm{DP}=$ desvio-padrão; $\mathrm{CV}=$ coeficiente de variação.

Para estimação dos componentes de (co)variância e predição dos valores genéticos, utilizou-se o programa multiple trait derivative-free restricted maximum likelihood (MTDFREML), descrito por Boldman et al. (1995), que aplica o método de máxima verossimilhança restrita, com algoritmos livres de derivadas, sob modelo animal, com inclusão da matriz de parentesco. Esse programa utiliza o SPARSPAK (George et al., 1980), que permite aumentar a capacidade de trabalho dos computadores, em termos computacionais.

Os critérios de convergência adotados foram: a - valor da predição de variância do simplex para localizar o mínimo de $-2 \log 1$ (em que 1 = função de máxima verossimilhança) $10^{-6} \mathrm{em}$ modelos com múltiplos parâmetros; e b - não haver alteração no valor das estimativas do simplex $-2 \quad \log$ de verossimilhança $10^{-9}$ em quatro repetições sucessivas. A cada convergência, o programa foi reiniciado, usando os valores obtidos na análise anterior como valores iniciais por quatro repetições sucessivas. Os valores genéticos foram preditos a partir desses componentes de variância.

O modelo estatístico proposto considerou o efeito fixo de GC, a idade da vaca ao parto como covariável (efeito linear e quadrático) e os efeitos aleatórios genético aditivo direto e genético aditivo materno. Nesse modelo, os pesos foram considerados como características distintas em cada região.

Os efeitos da interação genótipo $\times$ ambiente foram obtidos pelas correlações genéticas entre cada peso, considerado como característica distinta nas várias regiões.

Os dados foram analisados de duas formas, ora considerando-se uma característica por vez (análise unicaracterística) ora considerando-se duas características (análise bicaracterística). 
Em termos matriciais, o modelo foi:

$y=X b+Z u+W m+e$, em que:

$\mathrm{y}=$ vetor de observações de características medidas nos indivíduos;

$\mathrm{X}, \mathrm{Z}$ e $\mathrm{W}=$ matrizes de incidência dos efeitos fixos e aleatórios;

$\mathrm{b}=$ vetor de efeitos fixos incluindo $\mathrm{GC}$, região e a idade da vaca ao parto como covariável;

$\mathrm{u}=$ vetor dos efeitos aleatórios de valores genéticos aditivos diretos do animal;

$\mathrm{m}=$ vetor dos efeitos aleatórios de valores genéticos maternos;

$\mathrm{e}_{\mathrm{i}}=$ vetor dos erros aleatórios associados a cada observação.

As seguintes premissas foram adotadas:

$\mathrm{E}(\mathrm{y})=\mathrm{X} \beta ; \mathrm{E}(\mathrm{u})=0 ; \mathrm{E}(\mathrm{m})=0$;

$\operatorname{Cov}(\mathrm{u}, \mathrm{m})=\mathrm{Z}^{\prime} \mathrm{R}^{-1} \mathrm{~W} ; \operatorname{Cov}(\mathrm{u}, \mathrm{e})=0$;

$\operatorname{Cov}(\mathrm{m}, \mathrm{e})=0$;

$\mathrm{V}(\mathrm{y})=\mathrm{Z}$ G Z' + W M W' + 2 Z' G W + R1;

$\mathrm{V}(\mathrm{u})=\mathrm{G}$ matriz não singular conhecida das variâncias e covariâncias dos efeitos em u;

$\mathrm{V}(\mathrm{m})=\mathrm{M}$ matriz não singular conhecida das variâncias e covariâncias dos efeitos em m;

$\mathrm{V}(\mathrm{e})=\mathrm{R} \cdot \sigma^{2}$, em que $\mathrm{R}$ é a matriz não singular conhecida das variâncias e covariâncias dos efeitos residuais, admitindo-se que $\mathrm{V}(\mathrm{e})=\mathrm{I} \sigma_{\mathrm{e}}^{2}$.

Os vetores $\beta$, u e m são associados às observações do vetor $\mathrm{y}$ por meio das matrizes de incidência $X$, $\mathrm{Z}$ e $\mathrm{W}$, respectivamente. $\mathrm{O}$ sistema de equações do modelo misto (MME), desenvolvido por Henderson (1975) sob modelo animal, utilizado para estimação de efeitos fixos e predição dos aleatórios, está apresentado a seguir:

$$
\begin{aligned}
& {\left[\begin{array}{ccc}
X^{\prime} R^{-1} X & X^{\prime} R^{-1} Z & X^{\prime} R^{-1} W \\
Z^{\prime} R^{-1} X & Z^{\prime} R^{-1} Z+k_{1} & Z^{\prime} R^{-1} W+k_{2} \\
W^{\prime} R^{-1} X & W^{\prime} R^{-1} Z+k_{2} & W R^{-1} W+k_{3}
\end{array}\right]\left[\begin{array}{l}
\hat{b} \\
\hat{u} \\
\hat{m}
\end{array}\right]=\left[\begin{array}{c}
X R^{-1} y \\
Z^{\prime} R^{-1} y \\
W^{\prime} R^{-1} y
\end{array}\right]} \\
& \text { em que } k_{1}=A^{-1} \otimes \alpha_{1}, k_{2}=A^{-1} \otimes \alpha_{2}, k_{3}=A^{-1} \otimes \alpha_{3} \\
& \text { e }\left[\begin{array}{cc}
\alpha_{1} & \alpha_{2} \\
\alpha_{2} & \alpha_{3}
\end{array}\right]=\left[\begin{array}{ll}
g^{11} & g^{12} \\
g^{21} & g^{22}
\end{array}\right] ; \quad \text { sendo, }
\end{aligned}
$$

$$
G_{1}=\left[\begin{array}{ll}
g_{11} & g_{12} \\
g_{21} & g_{22}
\end{array}\right] \text { e } G^{-1}=\left[\begin{array}{ll}
g^{11} & g^{12} \\
g^{21} & g^{22}
\end{array}\right]
$$

Admitiu-se que a matriz de resíduos era diagonal em análises unicaracterísticas, sendo sua ordem equivalente ao número de animais, u é o vetor correspondente ao valor genético do desempenho produtivo dos animais, $\mathrm{m}$ é o vetor correspondente aos efeitos maternos dos animais e $\boldsymbol{e}$, o vetor de erros aleatórios temporários de ambiente, admitindo-se que u e $\boldsymbol{e}, \mathrm{m}$ e $\boldsymbol{e}$ não são correlacionados. Os dados de pedigree foram incluídos na matriz $\mathrm{A}$ do numerador dos coeficientes de parentesco. Algumas alterações nas dimensões e estrutura das equações foram realizadas nas análises bicaracterísticas, em razão de se considerarem duas características simultaneamente na análise.

Outra estatística utilizada para o estudo da interação genótipo $\times$ ambiente foi a correlação de posto que mede a associação entre os postos de pares de características. A magnitude da interação genótipo $\times$ ambiente para P205 e P365 foi também determinada pela correlação de posto entre valores genéticos preditos para cada par de combinação das regiões estudadas.

\section{RESULTADOS E DISCUSSÃO}

As estimativas de variância e covariância, herdabilidades e correlação genética entre os efeitos direto e materno dos pesos aos 205 (P205) e aos 365 (P365) dias de idade em cada uma das regiões são apresentadas nas Tab. 2 e 3, respectivamente.

As estimativas de (co)variância e componentes genéticos variaram muito em todas as regiões, para ambas as características, e podem ter ocorrido em razão do número diferente de informações em cada região. Na região Sudeste $(\mathrm{R} 2)$, em virtude do volume de informações disponíveis, foi obtida maior estimativa do componente $\sigma^{2}$ a do P205 $(70,432)$, e na região Sul (R1), menores estimativas do componente $\sigma^{2}$ a do P205 $(8,071)$ e do P365 $(25,034)$. 
Interação genótipo $\times$ ambiente...

Tabela 2. Variâncias genética aditiva direta $\left(\sigma_{\mathrm{a}}^{2}\right)$, genética aditiva materna $\left(\sigma_{\mathrm{m}}^{2}\right)$, fenotípica $\left(\sigma_{\mathrm{p}}^{2}\right)$, residual $\left(\sigma_{\mathrm{e}}^{2}\right)$, covariância entre os efeitos genético direto e materno $\left(\sigma_{\mathrm{am}}\right)$, herdabilidades direta $\left(\mathrm{h}_{\mathrm{a}}^{2}\right)$ e materna $\left(\mathrm{h}_{\mathrm{m}}^{2}\right)$ e correlação genética entre os efeitos direto e materno $\left(\mathrm{rg}_{\mathrm{am}}\right)$ obtidas em análise unicaracterística do peso aos 205 (P205) dias de idade, considerada como característica distinta em cada uma das regiões Sul (R1), Sudeste (R2), Centro-Oeste (R3) e Nordeste (R4)

\begin{tabular}{lcccccccc}
\hline Característica & $\sigma_{\mathrm{a}}^{2}$ & $\sigma_{\mathrm{m}}^{2}$ & $\sigma_{\mathrm{p}}^{2}$ & $\sigma_{\mathrm{e}}^{2}$ & $\sigma_{\mathrm{am}}$ & $\mathrm{h}_{\mathrm{a}}^{2}$ & $\mathrm{~h}_{\mathrm{m}}^{2}$ & $\mathrm{rg}_{\mathrm{am}}$ \\
\hline P205 (R1) & 8,071 & 115,123 & 371,978 & 218,303 & 30,481 & 0,02 & 0,31 & $1,00 \pm(2,16)$ \\
P205 (R2) & 70,432 & 79,728 & 417,67 & 280,902 & $-13,393$ & 0,17 & 0,19 & $-0,18 \pm(0,17)$ \\
P205 (R3) & 61,917 & 26,822 & 312,248 & 223,506 & 0,002 & 0,20 & 0,09 & 0,00 \\
P205 (R4) & 29,403 & 79,992 & 504,507 & 395,111 & 0,002 & 0,06 & 0,16 & 0,00 \\
\hline
\end{tabular}

Tabela 3. Variâncias genética aditiva direta $\left(\sigma_{a}^{2}\right)$, genética aditiva materna $\left(\sigma_{\mathrm{m}}^{2}\right)$, fenotípica $\left(\sigma_{\mathrm{p}}^{2}\right)$, residual $\left(\sigma_{\mathrm{e}}^{2}\right)$, covariância entre os efeitos genético direto e materno $\left(\sigma_{\mathrm{am}}\right)$, herdabilidades direta $\left(\mathrm{h}_{\mathrm{a}}^{2}\right)$ e materna $\left(\mathrm{h}_{\mathrm{m}}^{2}\right)$ e correlação genética entre os efeitos direto e materno $\left(\mathrm{rg}_{\mathrm{am}}\right)$ obtidas em análise unicaracterística do peso aos 365 (P365) dias de idade, considerada como característica distinta em cada uma das regiões Sul (R1), Sudeste (R2), Centro-Oeste (R3) e Nordeste (R4)

\begin{tabular}{lcccccccc}
\hline Característica & $\sigma_{\mathrm{a}}^{2}$ & $\sigma_{\mathrm{m}}^{2}$ & $\sigma_{\mathrm{p}}^{2}$ & $\sigma_{\mathrm{e}}^{2}$ & $\sigma_{\mathrm{am}}$ & $\mathrm{h}_{\mathrm{a}}^{2}$ & $\mathrm{~h}_{\mathrm{m}}^{2}$ & $\mathrm{rg}_{\mathrm{am}}$ \\
\hline P365 (R1) & 25,034 & 11,812 & 465,857 & 428,989 & 0,041 & 0,05 & 0,03 & 0,00 \\
P365 (R2) & 120,593 & 15,139 & 591,277 & 441,962 & 13,583 & 0,20 & 0,03 & $0,32 \pm(0,21)$ \\
P365 (R3) & 224,863 & 277,588 & 444,727 & 192,113 & $-249,838$ & 0,51 & 0,62 & $-1,00 \pm(0,97)$ \\
P365 (R4) & 110,965 & 34,265 & 753,985 & 557,941 & 50,814 & 0,15 & 0,05 & $0,82 \pm(0,39)$ \\
\hline
\end{tabular}

Os valores das correlações entre os efeitos direto e materno e seus respectivos erros-padrão para P205 e P365 dias de idade, em cada região, foram, respectivamente, iguais a: $1,00 \pm 2,16$ e 0 (R1), $0,18 \pm 0,17$ e $0,32 \pm 0,21$ (R2), 0 e $-1,00 \pm 0,97$ (R3), 0 e 0,82 $\pm 0,39$ (R4). Nota-se que essas estimativas foram erráticas e de baixa precisão.

Essas correlações foram negativas para P205 na região Sudeste e P365 na região Centro-Oeste, evidenciando antagonismo entre os efeitos direto e materno. Nesse caso, a seleção para incrementar o efeito materno poderia causar redução nos pesos dos animais. Mercadante e Lôbo (1997), Eler et al. (2000) e Ferraz Filho et al. (2002) também encontraram correlações negativas para P205 ($0,07,-0,24$ e $-0,42$, respectivamente), ao trabalharem com bovinos Nelore. Nas regiões Centro-Oeste e Nordeste, para a característica $\mathrm{P} 205$, e Sul, para P365, as $\mathrm{rg}_{\mathrm{am}}$ foram iguais a zero, que indicam dissociação entre os efeitos direto e materno. Valores positivos para essa correlação possibilitariam maior progresso genético na seleção (Willham, 1972). Mercadante e Lôbo
(1997) encontraram valor de correlação entre os efeitos direto e materno próximo a zero $(-0,01)$ para P205 em bovinos Nelore.

As estimativas de herdabilidade direta nas análises unicaracterísticas tanto para P205 quanto para P365 variaram entre regiões. Os valores obtidos para P205 e P365 foram 0,02 e 0,05 (R1), 0,17 e 0,20 (R2), 0,20 e 0,51 (R3) e 0,06 e 0,05 (R4). Estimativas semelhantes às obtidas para $\mathrm{P} 205$ na R2 $(0,17)$ e P365 $(0,15)$ na R4 foram também observadas por Ferraz Filho et al. (2002) que verificaram $\mathrm{h}^{2}{ }_{\mathrm{a}}$ iguais a $0,16(\mathrm{P} 205)$ e $0,17(\mathrm{P} 365)$ em animais Tabapuã.

As estimativas de herdabilidade materna nas análises unicaracterísticas foram semelhantes às encontradas por Ferreira et al. (2001) e Ribeiro et al. (2001) em bovinos Nelore. As estimativas de herdabilidade materna do P205 e P365, 0,31 e 0,03 na região Sul $(\mathrm{R} 1), 0,19$ e 0,03 na região Sudeste (R2) e 0,16 e 0,05 na região Nordeste (R4), indicam que $\mathrm{o}$ efeito materno exerceu maior influência no peso aos 205 dias de idade, pois, 
nessa fase, existe maior dependência do bezerro em relação às suas mães e menor importância para os pesos aos 365 dias de idade. Ferraz Filho et al. (2002), em análise do peso aos 365 dias de idade de animais Tabapuã, encontraram valor semelhante $(0,03)$ aos das regiões R1 e R2, enquanto Mercadante e Lôbo (1997) e Marcondes et al. (2002) encontraram, em animais Nelore, valores maiores do que os obtidos neste trabalho $(0,14 \mathrm{e}$ 0,10 , respectivamente).

As estimativas das variâncias e covariâncias genéticas aditivas direta e materna, fenotípicas e residuais de P205 e P365, consideradas características distintas nas diversas regiões, são apresentadas nas Tab. 4 e 5 , e 6 e 7, respectivamente.

Tabela 4. Estimativas de variâncias genéticas aditiva direta (a1-a2) e materna (m1-m2) (diagonal) e covariâncias (fora da diagonal), obtidas pela análise conjunta do peso aos 205 (P205) dias de idade, consideradas como características distintas nas diversas combinações das regiões Sul (R1), Sudeste (R2), Centro-Oeste (R3) e Nordeste (R4)

\begin{tabular}{|c|c|c|c|c|c|c|c|c|c|c|c|c|c|c|}
\hline & \multicolumn{4}{|c|}{ R1 e R2 } & & \multicolumn{4}{|c|}{ R1 e R3 } & & \multicolumn{4}{|c|}{ R1 eR4 } \\
\hline & a1 & a2 & $\mathrm{m} 1$ & $\mathrm{~m} 2$ & & a1 & $\mathrm{a} 2$ & $\mathrm{~m} 1$ & $\mathrm{~m} 2$ & & al & $\mathrm{a} 2$ & $\mathrm{~m} 1$ & $\mathrm{~m} 2$ \\
\hline a1 & 98,52 & & & & a1 & 89,10 & & & & a1 & 65,39 & & & \\
\hline $\mathrm{a} 2$ & 92,62 & 87,13 & & & $\mathrm{a} 2$ & 33,88 & 18,29 & & & $\mathrm{a} 2$ & $-33,35$ & 23,11 & & \\
\hline $\mathrm{m} 1$ & $-5,61$ & $-5,72$ & 116,25 & & $\mathrm{~m} 1$ & $-40,41$ & 9,66 & 67,65 & & $\mathrm{~m} 1$ & $-1,59$ & 22,56 & 78,01 & \\
\hline \multirow[t]{3}{*}{$\mathrm{m} 2$} & $-2,95$ & $-4,37$ & - & 67,20 & $\mathrm{~m} 2$ & 0,05 & $-0,14$ & $-0,13$ & 142,01 & $\mathrm{~m} 2$ & - & 2,64 & 1,06 & 143,50 \\
\hline & \multicolumn{4}{|c|}{ R2 e R3 } & & \multicolumn{4}{|c|}{ R2 e R4 } & & \multicolumn{4}{|c|}{ R3 e R4 } \\
\hline & al & a2 & $\mathrm{m} 1$ & $\mathrm{~m} 2$ & & a1 & $\mathrm{a} 2$ & $\mathrm{~m} 1$ & $\mathrm{~m} 2$ & & al & a2 & $\mathrm{ml}$ & $\mathrm{m} 2$ \\
\hline a1 & 67,88 & & & & a1 & 69,53 & & & & a1 & 38,52 & & & \\
\hline $\mathrm{a} 2$ & 64,30 & 63,48 & & & $\mathrm{a} 2$ & 18,90 & 19,90 & & & $\mathrm{a} 2$ & 50,43 & 66,05 & & \\
\hline $\mathrm{m} 1$ & $-13,24$ & 0,26 & 80,58 & & $\mathrm{~m} 1$ & $-13,05$ & $-1,20$ & 79,94 & & $\mathrm{~m} 1$ & $-0,02$ & 0,72 & 78,33 & \\
\hline $\mathrm{m} 2$ & $-0,25$ & - & 23,75 & 35,84 & $\mathrm{~m} 2$ & - & - & 82,13 & 87,45 & $\mathrm{~m} 2$ & - & - & 6,32 & 21,06 \\
\hline
\end{tabular}

Tabela 5. Estimativas de variâncias fenotípica (p1-p2) e residual (e1-e2) (diagonal) e covariâncias (fora da diagonal), obtidas pela análise conjunta do peso aos 205 (P205) dias de idade, consideradas como características distintas nas diversas combinações das regiões Sul (R1), Sudeste (R2), Centro-Oeste (R3) e Nordeste (R4)

\begin{tabular}{|c|c|c|c|c|c|c|c|c|c|c|c|c|c|c|}
\hline & \multicolumn{4}{|c|}{ R1 e R2 } & & \multicolumn{4}{|c|}{ R1 e R3 } & & \multicolumn{4}{|c|}{ R1 eR4 } \\
\hline & p1 & p2 & e1 & e2 & & p1 & p2 & el & $\mathrm{e} 2$ & & p1 & p2 & $\mathrm{e} 1$ & e2 \\
\hline pl & 388,80 & & & & p1 & 314,50 & & & & pl & 514,45 & & & \\
\hline p2 & 322,89 & 422,04 & & & p2 & $-70,05$ & 373,11 & & & p2 & $-296,77$ & 373,42 & & \\
\hline el & - & - & 179,64 & & e1 & - & - & 198,15 & & e1 & - & - & 372,64 & \\
\hline \multirow[t]{3}{*}{$\mathrm{e} 2$} & - & - & 221,08 & 272,09 & e2 & - & - & $-106,97$ & 212,94 & e2 & - & - & $-275,76$ & 204,07 \\
\hline & \multicolumn{4}{|c|}{ R2 e R3 } & & \multicolumn{4}{|c|}{ R2 e R4 } & & \multicolumn{4}{|c|}{ R3 e R4 } \\
\hline & p1 & p2 & el & e2 & & p1 & p2 & el & e2 & & p1 & p2 & e1 & e2 \\
\hline p1 & 417,34 & & & & p1 & 417,63 & & & & p1 & 506,68 & & & \\
\hline p2 & $-112,86$ & 317,10 & & & p2 & $-232,73$ & 503,39 & & & p2 & 353,52 & 312,65 & & \\
\hline e1 & - & - & 282,10 & & e1 & - & - & 281,19 & & e1 & - & - & 389,85 & \\
\hline e2 & - & - & $-200,92$ & 217,79 & $\mathrm{e} 2$ & - & - & $-333,15$ & 396,07 & e2 & - & - & 296,39 & 225,54 \\
\hline
\end{tabular}


Interação genótipo $\times$ ambiente...

Tabela 6. Estimativas de variâncias genéticas aditiva direta (a1-a2) e materna (m1-m2) (diagonal) e covariâncias (fora da diagonal), obtidas pela análise conjunta do peso aos 365 (P365) dias de idade, consideradas como características distintas nas diversas combinações das regiões Sul (R1), Sudeste (R2), Centro-Oeste (R3) e Nordeste (R4)

\begin{tabular}{|c|c|c|c|c|c|c|c|c|c|c|c|c|c|c|}
\hline & \multicolumn{4}{|c|}{ R1 e R2 } & & \multicolumn{4}{|c|}{ R1 e R3 } & & \multicolumn{4}{|c|}{ R1 eR4 } \\
\hline & al & $\mathrm{a} 2$ & $\mathrm{~m} 1$ & $\mathrm{~m} 2$ & & al & $\mathrm{a} 2$ & $\mathrm{ml}$ & $\mathrm{m} 2$ & & al & a2 & $\mathrm{m} 1$ & $\mathrm{~m} 2$ \\
\hline al & 116,67 & & & & al & 24,09 & & & & a1 & 42,96 & & & \\
\hline $\mathrm{a} 2$ & 53,32 & 25,04 & & & $\mathrm{a} 2$ & 53,98 & 121,89 & & & $\mathrm{a} 2$ & $-55,69$ & 136,20 & & \\
\hline $\mathrm{ml}$ & 4,88 & 2,12 & 26,18 & & $\mathrm{~m} 1$ & 2,33 & 3,49 & 11,19 & & $\mathrm{ml}$ & 0,46 & $-2,12$ & 10,02 & \\
\hline \multirow[t]{3}{*}{$\mathrm{m} 2$} & 0,01 & 0,04 & 28,80 & 32,09 & $\mathrm{~m} 2$ & 0,06 & 1,10 & 1,97 & 5,08 & $\mathrm{~m} 2$ & 28,05 & 16,14 & 17,17 & 66,42 \\
\hline & \multicolumn{4}{|c|}{ R2 e R3 } & & \multicolumn{4}{|c|}{ R2 e R4 } & & \multicolumn{4}{|c|}{ R3 e R4 } \\
\hline & al & a2 & $\mathrm{m} 1$ & $\mathrm{~m} 2$ & & al & a2 & $\mathrm{m} 1$ & $\mathrm{~m} 2$ & & al & a2 & $\mathrm{ml}$ & $\mathrm{m} 2$ \\
\hline al & 118,28 & & & & al & 139,43 & & & & a1 & 127,72 & & & \\
\hline a2 & 111,16 & 121,58 & & & $\mathrm{a} 2$ & 29,31 & 104,29 & & & $\mathrm{a} 2$ & 17,15 & 150,08 & & \\
\hline $\mathrm{ml}$ & $-10,07$ & $-24,11$ & 44,77 & & $\mathrm{~m} 1$ & 2,46 & 0,01 & 71,65 & & $\mathrm{~m} 1$ & $-130,46$ & 2,49 & 150,55 & \\
\hline $\mathrm{m} 2$ & $-0,01$ & 2,39 & 27,81 & 28,74 & $\mathrm{~m} 2$ & - & 0,01 & $-51,87$ & 37,57 & $\mathrm{~m} 2$ & $-0,03$ & $-0,29$ & $-30,83$ & 65,26 \\
\hline
\end{tabular}

Tabela 7. Estimativas de variâncias fenotípica (p1-p2) e residual (e1-e2) (diagonal) e covariâncias (fora da diagonal), obtidas pela análise conjunta do peso aos 365 (P365) dias de idade, consideradas como características distintas nas diversas combinações das regiões Sul (R1), Sudeste (R2), Centro-Oeste (R3) e Nordeste (R4)

\begin{tabular}{|c|c|c|c|c|c|c|c|c|c|c|c|c|c|c|}
\hline & \multicolumn{4}{|c|}{ R1 e R2 } & \multicolumn{5}{|c|}{ R1 e R3 } & \multicolumn{5}{|c|}{ R1 eR4 } \\
\hline & $\mathrm{p} 1$ & $\mathrm{p} 2$ & $\mathrm{e} 1$ & $\mathrm{e} 2$ & & $\mathrm{p} 1$ & $\mathrm{p} 2$ & e1 & $\mathrm{e} 2$ & & $\mathrm{p} 1$ & $\mathrm{p} 2$ & $\mathrm{e} 1$ & $\mathrm{e} 2$ \\
\hline p1 & 591,39 & & & & $\mathrm{p} 1$ & 464,83 & & & & $\mathrm{p} 1$ & 476,35 & & & \\
\hline $\mathrm{p} 2$ & 338,05 & 465,08 & & & $\mathrm{p} 2$ & 182,73 & 435,06 & & & $\mathrm{p} 2$ & $-135,38$ & 760,65 & & \\
\hline e1 & - & - & 443,66 & & e1 & - & - & 427,22 & & el & - & - & 422,90 & \\
\hline \multirow[t]{3}{*}{$\mathrm{e} 2$} & - & - & 254,81 & 407,91 & $\mathrm{e} 2$ & - & - & 122,08 & 306,95 & $\mathrm{e} 2$ & - & - & $-122,41$ & 541,88 \\
\hline & \multicolumn{4}{|c|}{ R2 e R3 } & & \multicolumn{4}{|c|}{ R2 e R4 } & & \multicolumn{4}{|c|}{ R3 e R4 } \\
\hline & $\mathrm{p} 1$ & $\mathrm{p} 2$ & $\mathrm{e} 1$ & $\mathrm{e} 2$ & & $\mathrm{p} 1$ & $\mathrm{p} 2$ & e1 & $\mathrm{e} 2$ & & $\mathrm{p} 1$ & $\mathrm{p} 2$ & $\mathrm{e} 1$ & $\mathrm{e} 2$ \\
\hline p1 & 445,11 & & & & $\mathrm{p} 1$ & 757,08 & & & & pl & 434,90 & & & \\
\hline $\mathrm{p} 2$ & 118,69 & 589,67 & & & $\mathrm{p} 2$ & 145,83 & 592,21 & & & $\mathrm{p} 2$ & $-407,27$ & 758,27 & & \\
\hline e1 & - & - & 292,13 & & e1 & - & - & 548,58 & & el & - & - & 287,10 & \\
\hline $\mathrm{e} 2$ & - & - & 245,60 & 436,95 & $\mathrm{e} 2$ & - & - & 62,12 & 439,79 & $\mathrm{e} 2$ & - & - & $-394,83$ & 542,98 \\
\hline
\end{tabular}

Em geral, ocorreu ampla variação nas estimativas de variâncias e covariâncias genéticas direta, materna e fenotípica tanto para o P205 quanto para o P365 em análises conjuntas, considerando-se cada uma das características como característica distinta nas combinações de pares de regiões estudadas. Isto se deveu em razão da expressiva variação no número de informações disponíveis em cada região. Nota-se isto quando as poucas observações disponíveis nas regiões Sul e CentroOeste são cotejadas com o número de informações disponíveis na região Sudeste.

As estimativas de herdabilidade e correlações genéticas entre P205 e P365, quando consideradas características distintas nas diversas regiões, obtidas em análises bicaracterísticas, são apresentadas nas Tab. 8 e 9, respectivamente.

Tabela 8. Estimativas de herdabilidade direta para as características $1\left(\mathrm{~h}_{\mathrm{a}}^{2} 1\right)$ e $2\left(\mathrm{~h}^{2}{ }_{\mathrm{a}}\right)$, herdabilidade materna para as características $1\left(h^{2}{ }_{m}\right)$ e $2\left(h_{m}^{2} 2\right)$, e correlações genéticas dos efeitos genéticos diretos dos pesos aos 205 (P205) dias de idade, consideradas como características distintas nas diversas combinações das regiões Sul (R1), Sudeste (R2), Centro-Oeste (R3) e Nordeste (R4)

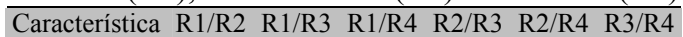

\begin{tabular}{lcccccc}
$\mathrm{h}^{2}{ }_{\mathrm{a}} 1$ & 0,25 & 0,28 & 0,13 & 0,16 & 0,17 & 0,08 \\
$\mathrm{~h}^{2}{ }_{\mathrm{a}} 2$ & 0,21 & 0,05 & 0,06 & 0,20 & 0,04 & 0,21 \\
$\mathrm{~h}^{2}{ }_{\mathrm{m}} 1$ & 0,30 & 0,22 & 0,15 & 0,19 & 0,19 & 0,15 \\
$\mathrm{~h}^{2}{ }_{\mathrm{m}} 2$ & 0,16 & 0,38 & 0,38 & 0,11 & 0,17 & 0,07 \\
$\mathrm{Rg}$ & 1,00 & 0,84 & $-0,86$ & 0,98 & 0,51 & 1,00 \\
\hline
\end{tabular}


Tabela 9. Estimativas de herdabilidade direta para as características $1\left(\mathrm{~h}_{\mathrm{a}}^{2} 1\right)$ e $2\left(\mathrm{~h}_{\mathrm{a}}^{2} 2\right)$, herdabilidade materna para as características $1\left(\mathrm{~h}_{\mathrm{m}}^{2} 1\right)$ e $2\left(\mathrm{~h}_{\mathrm{m}}^{2} 2\right)$, e correlações genéticas dos efeitos genéticos diretos dos pesos aos 365 (P365) dias de idade consideradas como características distintas nas diversas combinações das regiões Sul (R1), Sudeste (R2), Centro-Oeste (R3) e Nordeste (R4) \begin{tabular}{lllll}
\hline Característica R1/R2 R1/R3 R1/R4 R2/R3 R2/R4 R3/R4 \\
\hline her
\end{tabular} \begin{tabular}{lllllll}
\hline $\mathrm{h}^{2}{ }_{\mathrm{a}} 1$ & 0,20 & 0,05 & 0,09 & 0,27 & 0,17 & 0,29
\end{tabular} $\begin{array}{lllllll}\mathrm{h}^{2}{ }_{\mathrm{a}} 2 & 0,05 & 0,28 & 0,18 & 0,21 & 0,21 & 0,20\end{array}$ $\begin{array}{lllllll}\mathrm{h}^{2}{ }_{\mathrm{m}} 1 & 0,04 & 0,02 & 0,02 & 0,10 & 0,06 & 0,35\end{array}$

$\begin{array}{lllllll}\mathrm{h}^{2}{ }_{\mathrm{m}} 2 & 0,07 & 0,01 & 0,09 & 0,05 & 0,05 & 0,09\end{array}$

\begin{tabular}{lllllll}
$\mathrm{Rg}$ & 0,99 & 0,99 & $-0,73$ & 0,93 & 0,45 & 0,12 \\
\hline
\end{tabular}

Quando se consideram apenas dois ambientes, a interação genótipo $\mathrm{x}$ ambiente pode ser avaliada pela magnitude da correlação genética entre os pares de características, consideradas distintas nos dois ambientes, conforme sugeriu Falconer (1952), e utilizada por diversos autores (Notter et al., 1992; Meyer, 1995; Ferreira et al., 2001).

As correlações genéticas encontradas para P205 e P365, ambos considerados características distintas nas diversas regiões, foram 1,00 e $0,99,0,84$ e $0,99,-0,86$ e $-0,73,0,98$ e $0,93,0,51$ e $0,45,1,00$ e 0,12 para R1 e R2, R1 e R3, R1 e R4, R2 e R3, R2 e R4 e R3 e R4, respectivamente. Esses resultados indicam que, na desmama (P205), o efeito da interação foi observado somente nas combinações que envolveram a região Nordeste (R4) e as regiões Sul (R1) e Sudeste (R2). Para P365, o efeito da interação foi evidenciado em todas as combinações que envolveram a região Nordeste, com destaque entre as regiões R1 e R4 $(-0,73)$.

Tess et al. (1979), Koger et al. (1979) e Bertrand et al. (1985) encontraram evidências de interação genótipo $\mathrm{x}$ ambiente ao trabalharem com peso à desmama de animais Hereford e Simental, em diferentes regiões dos EUA. Resultados semelhantes foram também encontrados por Notter et al. (1992), ao analisarem o peso à desmama em diversos rebanhos na Austrália. Os autores afirmaram que as correlações genéticas observadas variaram de 0,52 a 0,61 . Ferreira (1999) observou correlação genética de 0,53 para peso pós- desmama, ao analisar dados de duas fazendas de regiões distintas no estado de Minas Gerais.

As altas correlações observadas nas Tab. 8 e 9, próximas a um, mesmo considerando o número de informações nas regiões, indicam que os mesmos grupos de genes são responsáveis pela expressão das duas características estudadas, com exceção dos pesos aos 205 dias de idade nas regiões Sul/Nordeste e Sudeste/Nordeste. Para o peso 365 dias de idade, isso ocorreu para todo par de combinação que envolveu a região Nordeste, o que indica, portanto, a existência da interação genótipo-ambiente.

De modo geral, as estimativas de herdabilidade direta do peso aos 205 dias de idade em análises bicaracterísticas (Tab. 8) foram maiores do que as encontradas em análises unicaracterísticas (Tab. 2). As maiores estimativas de $\mathrm{h}^{2}$ do peso aos 365 dias (Tab. 9) foram encontradas para as regiões R1/R3, R2/R3 e R3/R4. As demais estimativas de $h^{2}$ nessas análises bicaracterísticas foram inferiores às encontradas em análises unicaracterísticas (Tab. 3). Isso provavelmente aconteceu em razão da alteração no número de observações quando se considerar mais de uma característica na análise.

As correlações de posto entre os valores genéticos dos reprodutores com filhos em pelo menos três regiões, obtidas em análise bicaracterística, dos pesos aos 205 e aos 365 dias de idade, são apresentadas na Tab. 10.

Tabela 10. Correlação de posto entre os valores genéticos dos touros, obtida a partir de análises bicaracterísticas entre o par de combinação das regiões Sul (R1), Sudeste (R2), Centro-Oeste (R3) e Nordeste(R4)

\begin{tabular}{lcc}
\hline Combinação & \multicolumn{2}{c}{ Correlação de posto } \\
\cline { 2 - 3 } de regiões & P205 & P365 \\
\hline R1R2 & 0,999 & 0,999 \\
R1R3 & 0,937 & 0,999 \\
R1R4 & $\mathbf{- 0 , 8 1 1}$ & $\mathbf{- 0 , 5 1}$ \\
R2R3 & 0,979 & 0,993 \\
R2R4 & 0,939 & $\mathbf{0 , 7 6 5}$ \\
R3R4 & 0,989 & $\mathbf{0 , 5 3 2}$ \\
\hline
\end{tabular}


Os resultados das correlações de posto, ao se considerar P205 e P365 como características distintas nas diversas regiões, indicam que os reprodutores, em análise envolvendo pares de região, foram ordenados (geneticamente) de forma semelhante quando se consideraram as combinações das regiões Sul/Sudeste, Sul/CentroOeste e Sudeste/Centro-Oeste. Entretanto, isto não ocorreu quando se considerou a combinação Sul/Nordeste, cujos resultados indicaram altas correlações negativas $-0,811$ (P205) e $-0,51$ (P365).

Quando se considerou P205 dias de idade para a combinação Sul/Nordeste, o valor da correlação, $-0,811$, mostra que a classificação de um reprodutor em uma região seria diferente da realizada em outra região. Quando se considerou o peso aos 365 dias de idade, as correlações de posto, que envolveram combinações da região Nordeste com as demais regiões, apresentaram valores de 0,51 (Sul/Nordeste), 0,765 (Sudeste/Nordeste) e 0,532 (Centro-Oeste/Nordeste). Esse resultado reforça a idéia de que os reprodutores deveriam ser testados por região, principalmente quando se considera a região Nordeste. As grandes diferenças climáticas e nutricionais dos animais entre as regiões são responsáveis pelos diferentes desempenhos em cada região.

Tess et al. (1979) verificaram mudanças na classificação dos animais quando os dados foram analisados por um modelo contendo efeitos de touro $\mathrm{x}$ região e touro $\mathrm{x}$ rebanho/região para peso ao nascer e desmame, respectivamente. Bertrand et al. (1987) encontraram resultados semelhantes, com maior mudança de classificação entre GC dentro de região, e menor quando medida entre regiões. Buchanan e Nielsen (1979) também observaram efeitos das interações touros $\times$ região, touro $\times$ rebanho/região e touro $\mathrm{x}$ GC/região sobre a ordem de classificação dos animais entre as regiões, rebanhos e GC dentro de região, quando foram analisados os pesos ao desmame e aos 365 dias de idade dos animais.

A ordenação dos touros, realizada por região, indicou que, dos 20 animais com maior valor genético na região Sul, para peso aos 205 dias de idade, apenas 15, 14 e 15 foram os melhores nas regiões Sudeste, Centro-Oeste e Nordeste, respectivamente. Quando consideraram os valores genéticos do peso aos 365 dias de idade, apenas 15 , 13 e 10 , dos 20 com maior valor genético da região Sul, entraram na lista dos 20 melhores animais nas regiões Sudeste, Centro-Oeste e Nordeste, respectivamente.

O efeito da interação genótipo $\times$ ambiente sobre $o$ P365 indicou que reprodutores com melhores resultados nas regiões Sul, Sudeste e Centro-Oeste não apresentaram o mesmo desempenho na região Nordeste.

\section{CONCLUSÕES}

Os altos valores das herdabilidades maternas para P205 sugerem que o efeito materno deve ser incluído nos modelos de avaliação genética em razão da sua expressiva participação nessa fase. As baixas correlações genéticas e os valores das correlações de ordem indicam que a interação genótipo $\mathrm{x}$ ambiente é importante para P365 quando se consideram as combinações envolvendo a região Nordeste com as demais regiões. Reprodutores com melhores resultados nas regiões Sul, Sudeste e Centro-Oeste não apresentam o mesmo desempenho na região Nordeste.

\section{REFERÊNCIAS BIBLIOGRÁFICAS}

BERTRAND, J.K.; BERGER, P.J.; WILHAM, R.L. Sire $x$ environment interactions in beef cattle weaning weight field data. J. Anim. Sci., v.60, p.1397-1402, 1985.

BERTRAND, J.K.; HOUGH, J.D.; BENYSHEK, L.L. Sire $\mathrm{x}$ environment interactions and genetic correlations of sire progeny performance across regions in dam-adjusted field data. J. Anim. Sci., v.64, p.77-82, 1987.

BOLDMAN, K.G.; KRIESE, L.A.; VAN VLECK, L.D. et al. A manual for use of MTDFREML : a set of programs to obtain estimates of variance and covariance. Lincoln : Agricultural Research Service, 1995. 120p. (DRAFT). 
BUCHANAN, D.S.; NIELSEN, M.K.. Sire by environment interactions in beef cattle field data. J. Anim. Sci., v.48, p.307-312, 1979.

ELER, J.P.; FERRAZ, J.B.S.; GOLDEN, B.L et al. Influência da interação touro $\mathrm{x}$ rebanho na estimação da correlação entre efeitos genético direto e materno em bovinos da raça Nelore. Rev. Bras. Zootec., v. 29, p. 1642-1648, 2000.

FALCONER, D.S. The problem of environment and selection. Am. Nat., v.86, p.293-298, 1952.

FERRAZ FILHO, P.B.; RAMOS, A.A.; SILVA, L.O.C. et al. Tendência genética dos efeitos direto e materno sobre os pesos à desmama e pósdesmama de bovinos da raça Tabapuã. Rev. Bras. Zootec., v.31, p.635-640, 2002.

FERREIRA, V.C.P.; PENNA, V.M.; BERGMANN, J.A.G. et al. Interação genótipoambiente em algumas características produtivas de gado de corte no Brasil. Arq. Bras. Med. Vet. Zootec., v.53, p.385-392, 2001.

GEORGE, A.; LIU, J.W.H. User's guide for SPARSPAK: Waterloo Sparse Linear Equations Packages. Department of Computer Science, University of Waterloo, Waterloo, Ontario, Canadá, 1980. 151p.

HAMMOND, J. Animal breeding in relation to nutrition and environment conditions. J. Anim. Sci., v.22, p.195-213, 1947.

HENDERSON, C.R. 1975. Best linear unbiased prediction under a selection model. Biometrics 31:423.

KOGER, M.; BURNS, W.C.; PAHNISH, O.F. et al. Genotype by environment interactions in Hereford cattle: I. Reproductive traits. J. Anim. Sci., v.49, p.396-402, 1979.
MARCONDES, C.R.; GAVIO, D.; BITTENCOURT, T.C.C. et al. Estudos de modelos alternativos de componentes de (co)variância e precisão de dos valores genéticos de características de crescimento em bovinos da raça Nelore. Arq. Bras. Med. Vet. Zootec., v.54, p.93-99, 2002.

MERCADANTE, M.E.Z; LÔBO, R.B. Estimativas de (co)variância e parâmetros genéticos dos efeitos direto e materno de características de crescimento de fêmeas de um rebanho Nelore. Rev. Bras. Zootec., v.26, p. 1124$1133,1997$.

MEYER, K. Estimates of genetic parameters and breeding values for New Zealand and Australian Angus cattle. Aust. J. Agric. Res., v.46, p.12191229, 1995

NOTTER, D.R.; TIER, B.; MEYER, K. Sire x herd interactions for weaning weight in beef cattle. $J$. Anim. Sci., v.70, p.2359-2365, 1992.

RIBEIRO, M.N.; PIMENTA FILHO, E.C.; MARTINS, G.A. et al. Herdabilidade para efeitos diretos e maternos de características de crescimento de bovinos Nelore no Estado da Paraíba. Rev. Bras. Zootec., v.30, p.1224- 1227, 2001.

STATISTICAL Analysis System. Versão 6.12 para windows. Cary, NC: SAS Institute, 1997. 1290p.

TESS, M.W.; KRESS, D.D.; BURFENING, P.J. et al. Sire by environment interactions in Simentalsired calves. J. Anim. Sci., v.49, p.964-971, 1979.

WILLHAM, R.L. The role of maternal effects in animal breeding: III. Biometrical aspects in animals. J. Anim. Sci. v.35, p.1288-1293, 1972. 